\title{
Understanding ethical climate, moral distress, and burnout: a novel tool and a conceptual framework
}

\author{
Elizabeth Dzeng, ${ }^{1,2}$ J Randall Curtis ${ }^{3}$
}

'Department of Medicine, Division of Hospital Medicine, University of California, San Francisco, California, USA ${ }^{2}$ Department of Social and Behavioural Science, Sociology Program, University of California San Francisco, San Francisco, California, USA

${ }^{3}$ Division of Pulmonary, Critical Care, and Sleep Medicine, Department of Medicine, Cambia Palliative Care Center of Excellence, University of Washington, Seattle, Washington, USA

\section{Correspondence to} Dr Elizabeth Dzeng, University of California San Francisco, Division of Hospital Medicine, San Francisco CA 94143-0131, USA; liz.dzeng@ucsf.edu

Accepted 26 March 2018 Published Online First 18 April 2018

\section{SLinked}

http://dx.doi.org/10.1136/ bmjqs-2017-007390

Check for updates

To cite: Dzeng E, Curtis JR. BMJ Qual Saf

2018:27:766-770.
The pace of technological advancements in the intensive care unit (ICU) challenges clinicians' ability to manage ethical and decision-making challenges near the end of life. Modern medicine has advanced to the point where we can support multiple organ systems simultaneously and sustain life when the benefits of treatments to overall survival and quality of life are not always clear. Physiological and technological limits no longer always tell us when to stop, and clinicians and families are now forced to take over the role that was once played by nature to make decisions as to whether and when life-sustaining therapies should be withdrawn or withheld.

Unfortunately, we often do not do a very good job of making these tough decisions even when patients can participate in the discussion. To add to that challenge, patients often lack decision-making capacity during their ICU stay. Clinicians and families struggle to balance the inherently imperfect practice of substituted judgement with their own views on the best interests of the patient. Advance care planning can facilitate this process, but even with the best advance care planning, it is often a complex and uncertain process.

Recent evidence indicates that we are not negotiating this complex process well. ${ }^{12}$ Life-sustaining therapies are increasingly provided at the end of life in a way that confers no survival benefit and can cause harm. Older Americans with advanced dementia have experienced a doubling in the use of mechanical ventilation and a rise in ICU admission from $17 \%$ to $38 \%$ of those hospitalised in the last 30 days of life without substantial survival benefit. ${ }^{12}$ A recent cluster-randomised trial suggests that systematically increasing ICU admissions for older adults confers no mortality benefit, ${ }^{3}$ and other studies have shown a trend towards harm. ${ }^{45}$ Hospitals with higher frequency of ICU use have higher costs and greater use of invasive procedures without improvement in mortality. ${ }^{5}$ Overly aggressive, non-beneficial treatments are associated with reduced quality of life near death and lower perceptions of quality of care. ${ }^{6-10}$ Symptoms of post-traumatic stress disorder (PTSD) and depression are more likely among caregivers of patients who experience overly aggressive treatments. ${ }^{10-14}$ The ethical challenges inherent to considering the burdens and benefits of life-sustaining therapies near the end-of-life care, especially in the ICU, can be highly contentious and challenging. ${ }^{15} 16$

In the high-stakes environments of the ICU, clinician moral distress, originally defined by Jameton ${ }^{17}$ as the inability to act according to an individual's ethical beliefs due to structural or hierarchical constraints, can be particularly prevalent. ${ }^{18}$ While nurses have recognised the importance of ethical climate and moral distress for decades ${ }^{19}$ awareness of physician moral distress is a newer area of enquiry, ${ }^{20} 21$ perhaps reflecting the growing tensions that have arisen as a result of shifts in societal expectations surrounding autonomy and declines in physician power. ${ }^{22}$ While moral distress can occur in all areas of healthcare, perceived futile care is a particularly difficult and common ethical challenge in the ICU that frequently leads to clinician moral distress. ${ }^{20} 23$

Moral distress is an ethical root cause of clinician burnout, ${ }^{24}$ an urgent problem that affects more than half of US physicians. ${ }^{25}$ Clinician burnout is linked to poor clinician well-being, job dissatisfaction and job turnover. ${ }^{26-28}$ Burnout among medical students contributes to unprofessional behaviour and declines in 
empathy. ${ }^{29-31}$ Clinician burnout has negative effects on patient care, including reduced patient satisfaction, quality of care, patient rapport and patient safety, with higher rates of medical errors. ${ }^{32-34}$

Interventions to mitigate clinician moral distress and burnout tend to focus on the individual clinicians. ${ }^{35-38}$ Cultivating mindfulness and resilience is important and is a necessary step to alleviate moral distress, but must be integrated with interventions that target broader cultural norms that influence clinician behaviour and integrity. Although some have described the need to consider organisational and systems factors, little is known about the precise systemic inflection points and levers that influence moral distress and burnout. ${ }^{39}$ In particular, ethical climate, defined as 'individual perceptions of the organization that influences attitudes and behavior and serves as a reference for employee behavior', ${ }^{40}$ should be recognised as an important contributor that either alleviates or exacerbates moral distress.

In BMJ Quality \& Safety, Van den Bulcke and colleagues ${ }^{41}$ report the development and validation of the Ethical Decision-Making Climate Questionnaire (EDMCQ). This is an important step in allowing us to understand and subsequently design and evaluate interventions that modify an ICU's ethical climate to alleviate clinician moral distress and burnout and improve the patients' and families' experience. The authors developed this 35-question self-assessment instrument through a modified Delphi method that created a theoretical framework for ethical decision-making. The instrument was subsequently validated in 68 ICUs in 13 European countries and the USA. The ethical decision-making domains included within the EDMCQ are interdisciplinary collaboration and communication, leadership by physicians, and ethical climate. Although moral distress can manifest in many different ways, the EDMCQ allows us to specifically measure aspects of ethical climate as they relate to moral distress due to decision-making surrounding intensity of ICU care.

A clinician's decisions do not occur in a vacuum, but are instead embedded in a cultural milieu influenced by national policy, financial incentives, resource pressures, patient and family factors, and institutional leadership. Policy changes and systemic interventions can have unintended consequences that further disrupt this interconnected ecosystem. ${ }^{42}$ In a recent qualitative study, one of us (ED) found that an institution's ethical priority influenced the way physicians conceptualised autonomy and beneficence, which consequently influenced communication practices surrounding resuscitation decision-making near the end of life. ${ }^{43}$ The study also revealed the importance of systemic factors such as institutional cultural norms that contributed to inappropriately aggressive care at the end of life in the USA. ${ }^{44}$ This study and others highlight the importance of understanding and intervening on these institutional and ethical norms to mitigate overly aggressive care. ${ }^{45}$ We have previously hypothesised that interventions may be more effective if they target the attitudes, beliefs and culture that underlie communication practices rather than only the practices themselves. ${ }^{44}$

We propose a conceptual framework (figure 1) highlighting the importance of institutional culture and, in particular, of institutional ethical climate, which influences intensity of end-of-life care in the ICU and hospital setting, and subsequently contributes to clinician moral distress and burnout. We hypothesise that this causal pathway from ethical climate, to intensity of care, and subsequent moral distress and burnout result in substantive effects on patient and family outcomes including quality of life, experience of care, and presence of symptoms of PTSD, depression and prolonged bereavement. As such, the importance of the EDMCQ is its ability to measure, guide and evaluate

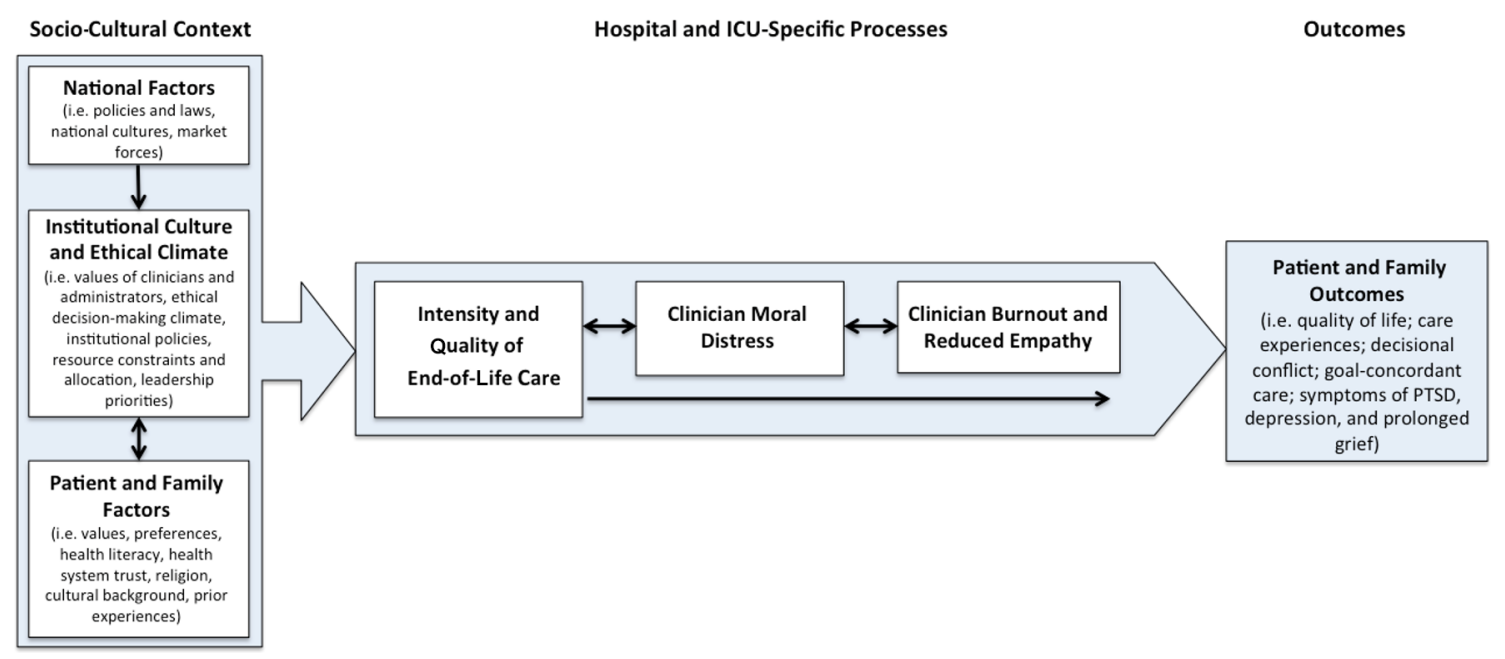

Figure 1 Conceptual model describing the relationship between ethical climate, clinician moral distress and burnout, and patient and family outcomes. The Ethical Decision-Making Climate Questionnaire focuses on institutional culture and ethical climate and its influence on institutional norms, which influence the intensity of end-of-life care. ICU, intensive care unit; PTSD, post-traumatic stress disorder. 
interventions on the ethical decision-making climate, providing the potential to improve patient, family and clinician outcomes.

Multiple qualitative and quantitative studies in the nursing literature have found that a positive ethical climate facilitated less moral distress among nurses. ${ }^{47-49}$ Given that in the ICU clinical decision-making is at the nexus of ethical challenges surrounding life-sustaining therapies near the end of life and a crucial moment for shifts in care trajectory, ethical decision-making climate is a key aspect of ICU ethical climate. The EDMCQ homes in on this critical facet of ethical climate that specifically links ethical climate with ICU treatment decisions at the end of life.

The EDMCQ scale is a valuable addition and update to existing ethical climate scales, the most prominent being the Olsen Hospital Ethical Climate Survey (HECS), which was designed and validated in the nursing literature. ${ }^{40}$ The HECS is a measure of ethical climate among nurses and focuses on factors related to nurses' relationships with actors within the hospital system, such as nurse peers, patients, managers, hospital administrators, and physicians. The EDMCQ focuses on physicians and nurses, as well as unit physician leadership, which all have profound effects on both ethical climate and ethical decision-making climate.

Given that physicians are often in positions of power in the hospital, involvement of and measurements that include physicians are important to an assessment of ethical culture. Studies using the HECS in physicians and nurses have shown that physicians generally rated ethical climate more positively than nurses, ${ }^{50}$ highlighting the power differential and affirming the importance of the EDMCQ's focus on interprofessional (particularly nurse-physician) trust, collaboration, and communication. In particular, the EDMCQ's emphasis on hearing the voices of all members of the team highlights the importance of the moral agency to speak up as being an important part of fostering positive ethical climates and alleviate moral distress. ${ }^{51} 52$ Furthermore, the EDMCQ's emphasis on interdisciplinary collaboration and communication is important as unit dysfunction and intrateam discordance exacerbate moral distress among members of the ICU team. ${ }^{18}$

The paper by Van den Bulcke and colleagues has some important strengths and weaknesses. In terms of strengths, this is a large and well-conducted study that included 3610 nurses and 1137 physicians working in 68 adult ICUs across Europe and the USA. The authors used a careful modified Delphi approach to develop a survey with strong face validity and they used rigorous and state-of-the-art exploratory and confirmatory factor analyses to determine the structure of the ethical decision-making climate concept. This report also has some important weaknesses. First, the 68 ICUs represent a convenience sample of ICUs that were willing to participate, although it is difficult to know how this might affect the findings. Second, the reports of ICU clinicians on their own ethical decision-making climate will undoubtedly be influenced by some degree of reporting bias, as well as by the fact that ICU clinicians may have limitations in their ability to observe the ethical norms in which they practise. Given these strengths and weaknesses, the EDMCQ is an important new measurement tool that should undergo additional validation as well as be compared with other methods to understand and assess the ethical decision-making climates in our ICUs.

We believe there has been insufficient recognition of moral distress as a key contributor to clinician burnout and poor well-being. Clinician burnout is a topic that has garnered significant interest and attention over the past several years. ${ }^{33} 5354$ In light of this crisis of clinician burnout, there is an urgent need to look to systemic and cultural root causes of burnout. One of the barriers to focusing on institutional culture and systems change is that it is difficult to measure culture. The EDMCQ helps advance the field by providing ways to measure ethical decision-making climate, thus facilitating future descriptive and intervention studies focusing on institutional and ethical culture to improve patient and family outcomes. Another benefit of the EDMCQ and other instruments that focus on the more humanistic aspects of medicine ${ }^{55}$ is that it draws attention to an institutional prioritisation of ethics and ethical climate among clinicians and administrators. ${ }^{56}$ The EDMCQ is an important step in improving the ethical decision-making climate of ICUs around intensity of end-of-life care and understanding its subsequent impact on the patient and family experience.

Contributor ED contributed to the conception of the paper. $\mathrm{ED}$ and JRC critically read and modified subsequent drafts and approved the final version.

Funding Research reported in this publication was supported by the National Center for Advancing Translational Sciences of the NIH under Award Number KL2TR001870. The content is solely the responsibility of the authors and does not necessarily represent the official views of the NIH.

Competing interests None declared.

Patient consent Not required.

Provenance and peer review Commissioned; internally peer reviewed.

(C) Article author(s) (or their employer(s) unless otherwise stated in the text of the article) 2018. All rights reserved. No commercial use is permitted unless otherwise expressly granted.

\section{REFERENCES}

1 Teno JM, Gozalo P, Khandelwal N, et al. Association of increasing use of mechanical ventilation among nursing home residents with advanced dementia and intensive care unit beds. JAMA Intern Med 2016;176:1809.

2 Teno JM, Gozalo PL, Bynum JP, et al. Change in end-of-life care for Medicare beneficiaries: site of death, place of care, and health care transitions in 2000, 2005, and 2009. JAMA 2013;309:470-7. 
3 Boumendil A, Angus DC, Guitonneau AL, et al. Variability of intensive care admission decisions for the very elderly. PLoS One 2012;7:e34387.

4 Guidet B, Leblanc G, Simon T, et al. Mortality among critically Ill elderly patients in France. J Am Med Assoc 2017;10.

5 Chang DW, Shapiro MF. Association between intensive care unit utilization during hospitalization and costs, use of invasive procedures, and mortality. JAMA Intern Med 2016;176:1492-9.

6 Huynh TN, Kleerup EC, Wiley JF, et al. The frequency and cost of treatment perceived to be futile in critical care. JAMA Intern Med 2013;173:1887-94.

7 Earle CC, Neville BA, Landrum MB, et al. Trends in the aggressiveness of cancer care near the end of life. J Clin Oncol 2004;22:315-21.

8 Prigerson HG, Bao Y, Shah MA, et al. Chemotherapy use, performance status, and quality of life at the end of life. JAMA Oncol 2015;1:778-84.

9 Wright AA, Keating NL, Ayanian JZ, et al. Family perspectives on aggressive cancer care near the end of life. JAMA 2016;315:284-92.

10 Wright AA, Zhang B, Ray A, et al. Associations between end-of-life discussions, patient mental health, medical care near death, and caregiver bereavement adjustment. JAMA 2008;300:1665-73.

11 van den Born-van Zanten SA, Dongelmans DA, DettlingIhnenfeldt $\mathrm{D}$, et al. Caregiver strain and posttraumatic stress symptoms of informal caregivers of intensive care unit survivors. Rehabil Psychol 2016;61:173-8.

12 Haines KJ, Denehy L, Skinner EH, et al. Psychosocial outcomes in informal caregivers of the critically ill: a systematic review. Crit Care Med 2015;43:1112-20.

13 Andresen M, Guic E, Orellana A, et al. Posttraumatic stress disorder symptoms in close relatives of intensive care unit patients: Prevalence data resemble that of earthquake survivors in Chile. J Crit Care 2015;30:1152.e7-1152.e11.

14 Probst DR, Gustin JL, Goodman LF, et al. ICU versus Non-ICU hospital death: family member complicated grief, posttraumatic stress, and depressive symptoms. J Palliat Med 2016;19:387-93.

15 Bosslet GT, Pope TM, Rubenfeld GD, et al. An Official ATS/ AACN/ACCP/ESICM/SCCM Policy Statement: Responding to Requests for Potentially Inappropriate Treatments in Intensive Care Units. Am J Respir Crit Care Med 2015;191:1318-30.

16 Schneiderman LJ, Jecker NS. Wrong medicine: doctors, patients, and futile treatment. 2nd edn. Baltimore: Johns Hopkins University Press, 2011.

17 Jameton A. Nursing practice: the ethical issues. Englewood Cliffs, NJ: Prentice Hall, 1984.

18 Bruce CR, Miller SM, Zimmerman JL. A qualitative study exploring moral distress in the ICU team: the importance of unit functionality and intrateam dynamics. Crit Care Med 2015;43:1-9.

19 Schluter J, Winch S, Holzhauser K, et al. Nurses' moral sensitivity and hospital ethical climate: a literature review. Nurs Ethics 2008;15:304-21.

20 Dzeng E, Colaianni A, Roland M, et al. Moral distress amongst American Physician trainees regarding futile treatments at the end of life: a qualitative study. J Gen Intern Med 2016;31:93-9.

21 Houston S, Casanova MA, Leveille M, et al. The intensity and frequency of moral distress among different healthcare disciplines. J Clin Ethics 2013;24:98-112.
22 Starr P. The social transformation of American medicine: the rise of a Sovereign profession and the making of a vast industry: Basic Books, 1982.

23 Mobley MJ, Rady MY, Verheijde JL, et al. The relationship between moral distress and perception of futile care in the critical care unit. Intensive Crit Care Nurs 2007;23:256-63.

24 Rushton $\mathrm{CH}$, Batcheller J, Schroeder K, et al. Burnout and resilience among nurses practicing in high-intensity settings. Am J Crit Care 2015;24:412-20.

25 Dyrbye L, Shanafelt T, Sinsky C, et al. Burnout among health care professionals: a call to explore and address this underrecognized threat to safe, high-quality care: National Academy of Medicine. https://nam.edu/burnout-amonghealth-care-professionals-a-call-to-explore-and-address-thisunderrecognized-threat-to-safe-high-quality-care/ (accessed 12 Mar 2018).

26 Shanafelt TD, Boone S, Tan L, et al. Burnout and satisfaction with work-life balance among US physicians relative to the general US population. Arch Intern Med 2012;172:1377-85.

27 Shanafelt T, Sloan J, Satele D, et al. Why do surgeons consider leaving practice? J Am Coll Surg 2011;212:421-2.

28 Shanafelt TD, Balch CM, Bechamps GJ, et al. Burnout and career satisfaction among American surgeons. Ann Surg 2009;250:107-15.

29 Brazeau CM, Schroeder R, Rovi S, et al. Relationships between medical student burnout, empathy, and professionalism climate. Acad Med 2010;85:S33-S36.

30 Thomas MR, Dyrbye LN, Huntington JL, et al. How do distress and well-being relate to medical student empathy? A multicenter study. J Gen Intern Med 2007;22:177-83.

31 Dyrbye LN, Massie FS, Eacker A, et al. Relationship between burnout and professional conduct and attitudes among US medical students. JAMA 2010;304:1173-80.

32 Dewa CS, Loong D, Bonato S, et al. The relationship between physician burnout and quality of healthcare in terms of safety and acceptability: a systematic review. BMJ Open 2017;7:e015141.

33 Dzau VJ, Kirch DG, Nasca TJ. To care is human - collectively confronting the clinician-burnout crisis. N Engl J Med Overseas Ed 2018;378:312-4.

34 Salyers MP, Bonfils KA, Luther L, et al. The relationship between professional burnout and quality and safety in healthcare: a meta-analysis. J Gen Intern Med 2017;32:475-82.

35 Rushton $\mathrm{CH}$. Principled moral outrage: an antidote to moral distress? AACN Adv Crit Care 2013;24:82-9.

36 Rushton CH, Sellers DE, Heller KS, et al. Impact of a contemplative end-of-life training program: being with dying. Palliat Support Care 2009;7:405-14.

37 Rushton CH, Kaszniak AW, Halifax JS. Addressing moral distress: application of a framework to palliative care practice. J Palliat Med 2013;16:1080-8.

38 Rushton $\mathrm{CH}$, Reder E, Hall B, et al. Interdisciplinary interventions to improve pediatric palliative care and reduce health care professional suffering. J Palliat Med 2006;9:922-33.

39 Back AL, Steinhauser KE, Kamal AH, et al. Building Resilience for Palliative Care Clinicians: An Approach to Burnout Prevention Based on Individual Skills and Workplace Factors. J Pain Symptom Manage 2016;52:284-91.

40 Olson LL. Hospital nurses' perceptions of the ethical climate of their work setting. Image J Nurs Sch 1998;30:345-9. 
41 Van den Bulcke B, Piers R, Jensen HI, et al. Ethical decisionmaking climate in the ICU: theoretical framework and validation of a self-assessment tool. BMJ Qual Saf 2018:1-9.

42 Kaufman S. And a time to die: how American hospitals shape the end of life. 1st edn. Chicago: University of Chicago Press, 2006.

43 Dzeng E, Colaianni A, Roland M, et al. Influence of institutional culture and policies on do-not-resuscitate decision making at the end of life. JAMA Intern Med 2015;175:812-9.

44 Dzeng E, Dohan D, Curtis JR, et al. Homing in on the social: system-level influences on overly aggressive treatments at the end of life. J Pain Symptom Manage 2018;55:282-9.

45 Barnato AE, Tate JA, Rodriguez KL, et al. Norms of decision making in the ICU: a case study of two academic medical centers at the extremes of end-of-life treatment intensity. Intensive Care Med 2012;38:1886-96.

46 Kelley AS, Bollens-Lund E, Covinsky KE, et al. Prospective identification of patients at risk for unwarranted variation in treatment. J Palliat Med 2018;21:44-54.

47 Corley MC, Minick P, Elswick RK, et al. Nurse moral distress and ethical work environment. Nurs Ethics 2005;12:381-90.

48 Pauly B, Varcoe C, Storch J, et al. Registered nurses' perceptions of moral distress and ethical climate. Nurs Ethics 2009; 16:561-73.
49 Atabay G, Çangarli BG, Penbek Ş. Impact of ethical climate on moral distress revisited: multidimensional view. Nurs Ethics 2015;22:1-14.

50 Bartholdson C, Sandeberg MA, Lützén K, et al. Healthcare professionals' perceptions of the ethical climate in paediatric cancer care. Nurs Ethics 2016;23:877-88.

51 Yerramilli D. On cultivating the courage to speak up: the critical role of attendings in the moral development of physicians in training. Hastings Cent Rep 2014;44:30-2.

52 Robinson EM, Lee SM, Zollfrank A, et al. Enhancing moral agency: clinical ethics residency for nurses. Hastings Cent Rep 2014;44:12-20.

53 Bodenheimer T, Sinsky C. From triple to quadruple aim: care of the patient. Ann Fam Med 2014;12:573-6.

54 Moss M, Good VS, Gozal D, et al. A critical care societies collaborative statement: burnout syndrome in critical Care health-care professionals. A call for action. Am J Respir Crit Care Med 2016;194:106-13.

55 Beach MC, Topazian R, Chan KS, et al. Climate of respect evaluation in ICUs: development of an instrument (ICUCORE). Crit Care Med 2018;6.

56 Hafferty FW. Beyond curriculum reform: confronting medicine's hidden curriculum. Acad Med 1998;73:403-7. 